\title{
Influence of Scaler Tip Design on Intensity of Pain and Discomfort During Scaling
}

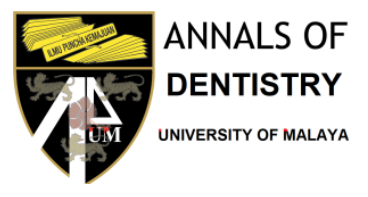

www.adum.edu.my

Ann Dent UM 2018, 25(2):36-42

Dol: 10.22452/adum.vol25no2.4

\author{
Mohamad Hanis Mat Nazri ${ }^{1}$, Mohd Fakhrul Ridwan Mohd Azmi ${ }^{1}$, Nor Adinar \\ Baharuddin $^{1}$, Roslan Saub ${ }^{2}$, Nur Ayman Abdul Hayei ${ }^{1}$, Rathna Devi Vaithilingam ${ }^{1 *}$
}

\section{KEYWORDS}

Slim scaler tips, conventional scaler tips, pain perception, scaling, gingivitis, VAS score

\begin{abstract}
To evaluate patient's subjective preferences to scaling with narrow probe-shaped EMS Perio Slim PS tips compared to conventional tip based on pain perception using the Visual Analogue Scale (VAS). Ten patients were treated using a piezoelectric ultrasonic device (EMS) and two different scaler tips representing a conventional scaler tip and a Perio Slim PS scaler tip in a split-mouth design. Pain was evaluated after 2 minutes scaling using Visual Analogue Scale (VAS). Data obtained was analysed by SPSS version 19.0 using Wilcoxon test. Pain assessment after treatment confirmed by the Visual Analogue Scale (VAS) showed that the Perio Slim PS scaler tip (maximum pain score: 4, minimum: 2) caused less pain than the conventional scaler tip (maximum: 8 , minimum: 3 ) ( $p$-value $=0.007$ ). Using Perio Slim PS scaler tip caused less pain and discomfort during scaling treatment when compared with conventional scaler tip.
\end{abstract}

\section{INTRODUCTION}

Calculus, which is a hard deposit comprised of mineralized bacterial plaque, is found on the tooth surface. Supragingival calculus can be found above the gingival margin while the calculus located below the gingival margin is known as subgingival calculus. Calculus predisposes to the development of periodontal disease by providing a retentive surface for bacterial dental biofilm [1]. It is the most widespread plaque-retentive factor. If these deposits are not removed, the bacteria that accumulates on them can cause inflammation of the gingiva with eventual bone resorption and tooth loss. Its removal is necessary for the longterm success of periodontal therapy [2]. Dentists spend a considerable amount of time to remove both supragingival and subgingival calculus which is routinely performed in the clinic [3]. Over the past few years, a patient centered approach to periodontal therapy has received more attention.

\footnotetext{
${ }^{1}$ Department of Restorative Dentistry, Faculty of Dentistry, University of Malaya, 50603, Kuala Lumpur.

${ }^{2}$ Department of Community Oral Health \& Clinical Prevention, Faculty of Dentistry, University of Malaya, 50603, Kuala Lumpur.

*Correspondence: rathna@um.edu.my
}

Patients often request for painless, less painful or less aggressive treatment methods. If two treatment methods with similar clinical outcomes and costs are offered, the patient preference will naturally be the less invasive or the less painful. Painful dental procedures may negatively affect patients. Thus, it will lead to patients avoiding further visits to the dentist [4]. In order to increase patient compliance, it is necessary to investigate aspects of this procedure, which affects the majority of the population in the world. Two common instrumentation methods used in removing bacterial plaque and calculus are the hand and power-driven instruments. Examples of hand instruments are sickle, curette, file, hoe and chisel. The most frequently used manual scaler is the curette type scalers. The Universal and Gracey curettes are the basic types of curettes. Gracey curettes have double-ended cutting edges which are site-specific and are used for root surface debridement as they are able to access deep and narrow pockets. Ultrasonic and sonic scalers are referred to as power-driven scalers. High vibrational energy generated in the oscillation generator is conducted to the scaler tip, causing vibrations to crush and remove calculus. Sonic and ultrasonic scalers vary in their efficiency in 
removing calculus from the tooth surfaces [5]. Sonic scalers are air-turbine units that operate at low frequencies ranging between 3000 and 8000 cycles per second (Cps). The tip movement is orbital. On the other hand, ultrasonic scalers are driven by generators which convert electrical energy into ultrasonic waves via piezoelectricity or magnetostriction and currently are available in two basic types which are piezoelectric and magnetostrictive scalers [6]. For magnetostrictive instruments, the tip vibrations are created by a resonating stack on the back of the insert. The vibrations generated range from 20 to more than $45 \mathrm{kHz}$ depending on the type of instrument. The instrument tip vibrates mostly in ellipsoidal, spatial vibrations which is unlikely to remove calculus actively in all directions. As for piezoelectric instruments, the vibrations are produced by oscillations of a quartz crystal in the handpiece. The vibration frequency ranges from 20 to $35 \mathrm{kHz}$. The tips mostly vibrate in linear motion and it is unlikely that all parts of the tips remove calculus to the same extent [6]. At the same time, the biofilm and calculus get disrupted by the cavitation effect around the scaler tip [7, 8]. Ultrasonic dental scalers are more commonly used in dental practice for scaling and removing stains as they help in reducing operating time and reduction of bacterial dental biofilm in periodontal pockets [9]. However, Gracey curettes are the instrument of choice for root surface debridement due to their narrow, site-specific tips which can access deep pockets. Recently, ultrasonic tips were modified to have smaller tips with longer working length to provide better access to deep probing sites which were more efficient in subgingival instrumentation [10]. An in vitro study has shown that a slim-line-styled tip of ultrasonic scaler may result in reducing pain intensity during supragingival calculus removal when compared with conventional ultrasonic device [11]. Thus far, no known study has compared the levels of discomfort and pain during ultrasonic debridement therapy using the following piezoelectric ultrasonic scaler inserts from the EMS Piezon ${ }^{\circledR}$, Switzerland i.e. (i) conventional scaler tip (FS-407) and (ii) Perio Slim PS scaler tip (DS-016A). Therefore, our study aimed to compare patients' perception of pain and discomfort using the Visual Analogue Scale (VAS) while performing scaling using these two insert tips. The results of this study may influence the selection of tips during scaling and root surface debridement by dentists using ultrasonic scalers.

\section{MATERIALS AND METHODS}

\section{Study design}

This pilot study was conducted using a randomized cross-over split mouth study model. Ethical approval has been obtained from the Medical Ethics Committee, Faculty of Dentistry, University of Malaya prior to conducting the study [DF RD1705/0022(U)].

\section{Subject recruitment}

The subjects were those who came to the Primary Care Unit, Faculty of Dentistry, University of Malaya for periodontal treatment. Those who fulfilled the inclusion and exclusion criteria were invited to participate in the study. As this was a pilot study, sample size calculation was not performed. A total of ten subjects were recruited for the study.

\section{Inclusion criteria}

1. Patients aged between 20 and 40 years old.

2. Patients with no systemic health condition.

3. Patients diagnosed with chronic gingivitis or mild chronic periodontitis with pocket depths of $3 \mathrm{~mm}$ to $5 \mathrm{~mm}$ (at least five sites with pocket depths of $4 \mathrm{~mm}$ )

4. Patients who have positive bleeding on probing in quadrant one and two.

5. Patients who have anterior maxillary teeth from teeth \# 13 to 23.

\section{Exclusion criteria}

1. Patients who are smokers

2. Patients who suffer from dentinal hypersensitivity involving one or more teeth in each quadrant.

3. Patients who have non-vital teeth, large restorations or crowns involving teeth \# 13-23.

4. Patients who suffer from any pulpitis, abscesses, cervical lesions or other acute dental infections requiring immediate treatment.

5. Patients who are on medication such as long term non-steroidal anti-inflammatory drug therapy.

6. Patients who are undergoing orthodontic treatment or using removable partial dentures involving teeth \# 13 - 23.

\section{Randomisation}

Subjects were randomly allotted into Group A and Group B based on order of recruitment. Randomisation was done using SPSS software. 
Predetermine recruitment numbers (1-10) were randomly allotted into group A or B using simple random sampling.

\section{Measurements}

\section{a) Questionnaire}

Questionnaire for patients comprised questions on sociodemographics (age, gender, ethnicity) and lifestyle habits. Participants were also asked questions on oral hygiene habits, include frequency of brushing, use of interdental cleaning and mouth rinse.

\section{b) Clinical measurement}

Following consent, patients underwent full periodontal examination. Periodontal parameters were charted from teeth \#13 to \#23 using William's probe (Hu-Friedy, Chicago, USA). The following periodontal parameters were charted:

I. Dental biofilm measurement using Visual plaque index (VPI) (Ainamo \& Bay, 1975)

Assessment of VPI was carried out at four sites of each tooth (mesiobuccal, mid-buccal, distobuccal and palatal/lingual surfaces) using dichotomous scoring system. The visible detection of plaque was marked as presence (1) or absence (0) by running a probe on the tooth surfaces.

$0=$ no visible plaque

1 = visible plaque

II. Gingival health measurement using Gingival Bleeding Index (GBI) (Ainamo \& Bay, 1975)

Assessment of $\mathrm{GBI}$ was carried out at four sites each tooth (mesiobuccal, mid-buccal, distobuccal and palatal/lingual surfaces) using the dichotomous scoring method. The assessment was considered as presence (1) if there is bleeding within ten seconds and absence (0) if there is no bleeding, after probing of the gingiva. William's probe was used for this bleeding assessment.

$0=$ no visible bleeding

$1=$ visible bleeding

III. Probing Pocket Depth (PPD)

PPD was measured from the gingival margin to the base of pocket. Measurement was carried out using a William's probe (Hu-Friedy, Chicago USA) with calibrated markings, by placing the probe parallel to the long axis of the tooth. Measurements closest to milimetre $(\mathrm{mm})$ were recorded. PPD were measured at six sites for teeth \#13 to \#23, which include mesiobuccal, mid-buccal, disto-buccal, mesiopalatal/lingual, mid-palatal/lingual and distopalatal/lingual surfaces.

IV. Recession (R)

$R$ was measured from the visible level of cemento-enamel junction (CEJ) (if present) to the gingival soft tissue margin. Measurement was carried out using a William's probe with calibrated markings, by placing the probe parallel to the long axis of the tooth. Measurements closest to millimetre $(\mathrm{mm})$ were recorded. $\mathrm{R}$ was measured at 6 sites per tooth for teeth \#13to \#23 which include mesiobuccal, mid-buccal, disto-buccal, mesiopalatal/lingual,mid-palatal/lingual and distopalatal/lingual surfaces.

V. Clinical Attachment Loss (CAL)

CAL was measured from the CEJ to the base of pocket. The level of CAL is the sum of PPD and R.

c) Pain level was measured using Visual Analogue Scale (VAS)

The VAS scale shown in Figure 1 consists of one line where the far-left end (scale 0 ) indicates no pain while the far-right end (scale 10) indicates the worst possible pain. Patient chose any number between these two ends that described best the pain they experienced during treatment.

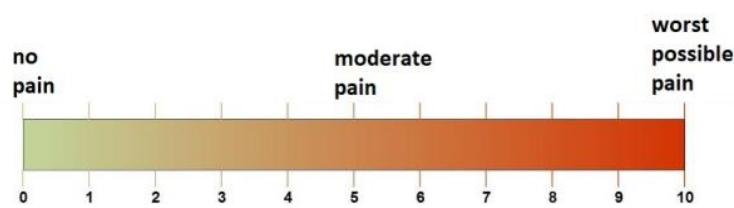

Figure 1. Visual Analogue Scale (VAS scale

\section{Data Collection}

The nature of the study was explained to all subjects and written informed consent was obtained. Questionnaire forms were then given to subjects to be filled prior to the intervention.

\section{Clinical intervention}

Flow chart of the clinical intervention is shown in Figure 2. Periodontal examination and scaling were performed by a periodontology postgraduate student of University Malaya. Parameters assessed were VPI, GBI, PPD and recession. Supra and subgingival scaling was only performed at teeth \#13 to \#23. Based on the randomization, Group A subjects were treated first with EMS Piezon Perio 
Slim PS scaler tip (DS-016A) at teeth \# 13-11 while Group B subjects were treated first with EMS Piezon conventional scaler tip (FS-407) at teeth \# 13-11.

Subjects were given an hour break before proceeding with scaling on the contralateral half sextant with the next scaler tip. This round the Group A was then treated with EMS Piezon ${ }^{\circledR}$ conventional scaler tip (FS-407) at teeth \#21-23 while Group B was treated with EMS Piezon ${ }^{\circledR}$ Perio Slim PS scaler tip (DS-016A) at teeth \# 21-23. Irrespective of calculus quantity, instrumentation was performed for two minutes with each device. Patients were blinded to the type of instruments used for both half sextants.

Power setting for both conventional scaler tips and perio slim PS scaler tips was set at medium setting for all patients as this was the recommended setting by the manufacturer for pain-sensitive patients and for supragingival maintenance-type treatment. During instrumentation, the instrument tips of both devices were always held parallel to the tooth's long axis. Depending on the halfsextant to be instrumented, the scaling (supra- and subgingival) was performed in a systematic method beginning from disto-buccal of tooth \#13 or tooth \#23 moving on to all buccal surfaces up to mesio-buccal of tooth \#11 or tooth \#21. The scaling then proceeded to mesio-palatal tooth \#11 or tooth \#21 and finally to all palatal surfaces up to the disto-buccal surface of tooth \#13 or tooth \#23. Immediately following calculus removal for each half sextant, subjects were given a VAS scale for pain to indicate their pain perception using the respective instrument. The VAS was graduated in the usual manner, i.e., from zero (no pain) to ten (unbearable pain). Information about the procedure and how to score VAS was explained to all patients prior to treatment. At the end of the study, subjects were given a full mouth scaling and polishing including removal of residual calculus that was still present on teeth \#13-23. The flow chart of the study is shown in Figure 2.

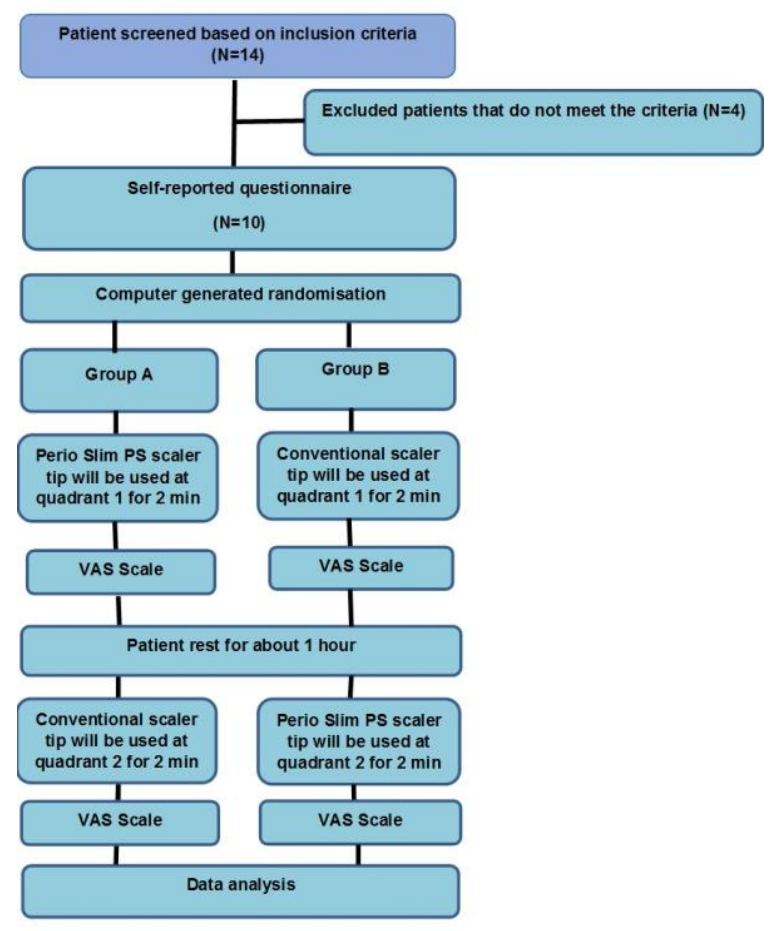

Figure 2. Flow chart of activity

\section{Data management and analysis:}

Data were analyzed using Statistical Package for Social Science Software Version 19.0 (SPSS). Due to the small sample size, non-parametric test was used. The baseline clinical parameters and the difference in the VAS scale after therapy between the 2 groups were compared using the Wilcoxon test. The level of significance was set at $\alpha=0.05$.

\section{RESULTS}

Demographic characteristics of participants are shown in Table 1. Since this is a pilot study and the sample size is too small for valid analysis, therefore descriptive analysis was performed. There were 6 men and 4 women. 
Table 1. Demographic characteristics of participants

\begin{tabular}{|l|l|c|c|}
\hline \multirow{2}{*}{ Characteristics } & \multicolumn{2}{c|}{ Group } \\
\cline { 3 - 4 } \multicolumn{2}{|c|}{} & $\begin{array}{c}\text { Group A } \\
\text { n=5 (\%) }\end{array}$ & $\begin{array}{c}\text { Group B } \\
\text { (n=5) }\end{array}$ \\
\hline \multirow{2}{*}{ Gender } & Male & $2(40)$ & $4(80)$ \\
\cline { 2 - 4 } & Female & $3(60)$ & $1(20)$ \\
\hline \multirow{2}{*}{ Ethnicity } & Malay & $4(80)$ & $5(100)$ \\
\cline { 2 - 4 } & Others & $1(20)$ & $0(0)$ \\
\hline \multirow{2}{*}{ Leve } & $20-30$ & $3(60)$ & $4(80)$ \\
\cline { 2 - 4 } & $30-40$ & $2(40)$ & $1(20)$ \\
\hline \multirow{2}{*}{ education } & Primary & $0(0)$ & $0(0)$ \\
\cline { 2 - 4 } & Secondary & $1(20)$ & $0(0)$ \\
\cline { 2 - 4 } & Tertiary & $4(80)$ & $5(100)$ \\
\hline
\end{tabular}

The participants' age ranged from 20-40 years with the majority being in the 20-30 years age range. There were more males than females in the control group. All participants were Malays except for one participant who was a native from Sabah. Majority of participants had tertiary education. Table 2 shows habits of the participants.

Table 2. Habits of participants

\begin{tabular}{|c|c|c|c|c|}
\hline \multicolumn{3}{|c|}{ Activity } & $\begin{array}{c}\text { Group } \\
\text { A }\end{array}$ & $\begin{array}{c}\text { Group } \\
\text { B }\end{array}$ \\
\hline \multirow{2}{*}{$\begin{array}{l}\text { Dental } \\
\text { Visit } \\
\mathrm{N}(\%) \\
\end{array}$} & \multicolumn{2}{|l|}{ Regular } & $1(20)$ & $2(40)$ \\
\hline & \multicolumn{2}{|l|}{ Irregular } & $480)$ & $3(60)$ \\
\hline \multirow{6}{*}{$\begin{array}{l}\text { Oral } \\
\text { Hygiene } \\
\text { Habit }\end{array}$} & \multirow{3}{*}{$\begin{array}{l}\text { Tooth } \\
\text { Brushing } \\
\text { Frequency }\end{array}$} & $<1 X /$ day & $0(0)$ & $0(0)$ \\
\hline & & $1 \mathrm{X} /$ day & $0(0)$ & $1(20)$ \\
\hline & & $>1 X /$ day & $5(100)$ & $4(80)$ \\
\hline & \multirow{3}{*}{$\begin{array}{l}\text { Interdental } \\
\text { cleaning }\end{array}$} & Flossing & $1(20)$ & $1(20)$ \\
\hline & & Toothpick & $0(0)$ & $3(60)$ \\
\hline & & $\begin{array}{l}\text { Interdent } \\
\text { al Brush }\end{array}$ & $0(0)$ & $0(0)$ \\
\hline & $\begin{array}{l}\text { Mouth- } \\
\text { rinse }\end{array}$ & & $2(40)$ & $0(0)$ \\
\hline \multicolumn{5}{|c|}{$\begin{array}{l}\text { Group A: Perio Slim PS scaler tip at Q1 followed by } \\
\text { Conventional scaler tip at Q2 } \\
\text { Group B: Conventional scaler tip at Q1 followed by Perio } \\
\text { Slim PS scaler tip at Q2 }\end{array}$} \\
\hline
\end{tabular}

Majority of participants were irregular dental attendees. In terms of oral hygiene habits, majority of participants brushed their teeth more than once a day. Interdental cleaning was only performed by 1 participant in the group $A$ while 4 participants in the group $B$ performed interdental cleaning. There were no significant differences for the periodontal clinical parameters at baseline between the treated teeth \#13-11 and \#21-23 as presented in Table 3.
Table 3. Baseline clinical parameters for treated teeth (tooth \# 13 to \#23)

\begin{tabular}{|c|c|c|c|}
\hline $\begin{array}{c}\text { Clinical } \\
\text { parameters }\end{array}$ & $\begin{array}{c}\text { Left } \\
(\mathbf{N = 1 0 )}\end{array}$ & $\begin{array}{c}\text { Right } \\
(\mathbf{N = 1 0})\end{array}$ & \multirow{2}{*}{$\mathbf{p}$-value } \\
\cline { 2 - 3 } & $\begin{array}{c}\text { Median } \\
(\text { IQR) }\end{array}$ & $\begin{array}{c}\text { Median } \\
(\text { IQR) }\end{array}$ & \\
\hline Mean VPI (\%) & $\begin{array}{c}51.7 \\
(0.14)\end{array}$ & $\begin{array}{c}48.3 \\
(0.26)\end{array}$ & 0.68 \\
\hline Mean GBI (\%) & $\begin{array}{c}53.3 \\
(13.15)\end{array}$ & $\begin{array}{c}58.3 \\
(13.03)\end{array}$ & 0.15 \\
\hline Mean PPD (mm) & $\begin{array}{c}2.7 \\
(0.17)\end{array}$ & $\begin{array}{c}2.7 \\
(0.22)\end{array}$ & 0.55 \\
\hline Mean CAL (mm) & 2.92 & 2.87 & 0.55 \\
& $(0.16)$ & $(0.23)$ & \\
\hline
\end{tabular}

VPI, Visible Plaque Index; GBI, Gingival Bleeding Index; PPD, Probing pocket depth; CAL, Clinical Attachment level; IQR: inter-quartile range

*p-value for Wilcoxon test

VAS measurement after therapy showed that Perio Slim PS scaler tip had a lower score as compared to the conventional scaler tip (Table 4). For the Perio Slim PS scaler tip, the average VAS value was 3.4 ( $S D \pm 0.7$ ), and for the conventional scaler tip, this was 5.7 (SD \pm 1.5 ). There were significant differences in perceived pain depending on scaler tip used as shown in Table 4 with $p$-value of 0.007 . From Figure 3, VAS scale scores for Perio Slim PS scaler tip ranged from 2 to 4 with score 4 chosen by 5 patients (50\%). The range of VAS scale scores for conventional scaler tip was from 3 to 8 with score 6 chosen by 3 patients (30\%).

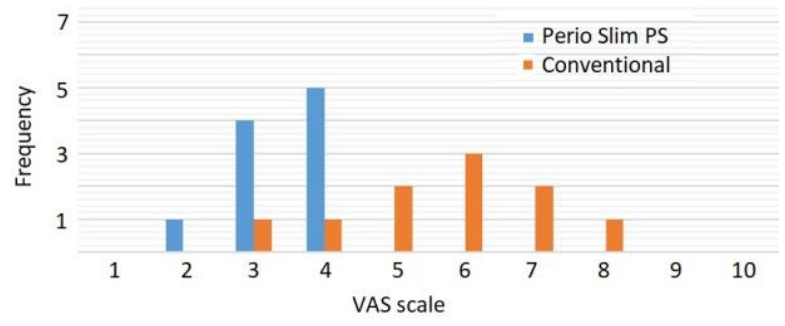

Figure 3. Frequency distribution of VAS scale for Perio Slim PS and conventional scaler tips

Table 4. VAS score after therapy for treated teeth (tooth \# 13 to \#23)

\begin{tabular}{|l|c|c|c|}
\hline \multirow{2}{*}{ Scaler tip } & \multicolumn{3}{|c|}{ VAS Score } \\
\cline { 2 - 4 } & $\begin{array}{c}\text { Left } \\
(\mathrm{N}=5) \\
\text { Median }\end{array}$ & $\begin{array}{c}\text { Right } \\
(\mathrm{N}=5) \\
\text { Median }\end{array}$ & $\begin{array}{c}\text { Total } \\
\mathrm{N}=10 \\
\text { Median }\end{array}$ \\
\hline Perio Slim PS & $3.6(0.55)$ & $3.2(0.837)$ & $3.4(0.7)$ \\
\hline Conventional & $5.2(1.8)$ & $6.2(1.1)$ & $5.7(1.5)$ \\
\hline p-value & & & 0.007 \\
\hline
\end{tabular}




\section{DISCUSSION}

The findings from this pilot study using VAS scores after scaling procedure showed that Perio Slim PS (DS-016A) ultrasonic scaler tips caused significantly less pain sensation compared to the Conventional (FS-407) ultrasonic scaler tips. This corroborates the findings from the study by Braun et al (2007) where they compared the pain intensity between a slim tip and a conventional tip. However, in their study, the time taken with each tip was not standardized. The demographic characteristics and habits of our participants did not have an effect on their disease status as both groups had similar levels of periodontal disease. Thus, our study demonstrates that within equally distributed periodontal conditions and time-frame, the Slimline tips caused significantly less pain than the conventional scaler tips.

Another study looking at pain during prophylaxis treatment compared a sonic and an ultrasonic scale [12]. However, this study used the piezoelectric ultrasonic scaler with maximum power settings and sonic scaler with no power adjustment possible. No difference could be observed between these two treatment devices when pain was measured using VAS. In the current study our comparisons between the different tips were controlled by the fact that the power setting used for all patients was standardized.

Piezoelectric tips which are slim-line-styled are designed to have a thin and very long body to access deep pockets and furcation area of posterior teeth. According to study by Pattison et al., (2003) [13], these adaptable thin piezoelectric tips are used to remove calculus with less burnishing when compared with the conventional scaler tip. The scaler tip that was used in this study was EMS Perio Slim PS which is long and thin with flat edges also provides better access to interproximal and subgingival areas. scaler tip that was used in this study was EMS Perio Slim PS which is long and thin with flat edges also provides better access to interproximal and subgingival areas.

A previous study has compared the pain intensities following periodontal therapy using different instruments such as the magnetostrictive scalers compared to conventional piezoelectric scalers and it was found that the magnetostrictive scalers caused less pain [14]. However, Jepsen, Ayna [15] observed that magnetostrictive device contributed to more severe root surface damage compared to piezoelectric scaler tips. They also reported that a significant increase in the aggressiveness to root dentin was seen for wide scaler tips as compared with narrow probe-shaped instruments. In our study, we have not assessed the effect of the 2 scaler tips on root surface damage and this should be assessed in a future study.

The current study used VAS scores to assess the pain intensity after therapy. However, in the study carried out by Braun et al (2007) to determine the pain intensity using ultrasonic scaler (piezoelectric slim-line-styled and conventional scaler tips) for supragingival calculus removal, the authors concluded that the usage of VAS alone was not sufficient as the ultrasonic vibration did not represent an exact temporally defined and irreversible peripheral stimulus. They found that assessing pain after scaling treatment using VAS had its limitations as the VAS scale could only be used to record a retrospective assessment of previously painful sensation and therefore a quick high intensity of pain might not be recorded correctly (Huskinsson 1983, Tammaro et al. 2000). They had suggested the additional use of the intermodal intensity comparisons whereby a manometer is used to determine the intensity of pain as VAS does not include time as a variable [11]. Inter-modal intensity comparison is used to increase the precision of pain intensity recorded. It was possible to correlate every single painful sensation to the exact treatment time. In the current study we were not able to perform this but future studies comparing pain intensities using these inserts should take this into account.

Many studies have looked at intensities of painful sensations during supra- and subgingival periodontal treatment using different powerdriven devices and scaler tip styles $[11,16]$. The strength of the current study lies in its design which was done to control various possible confounders. The power of the ultrasonic device used for all participants was standardized and set to medium setting (power setting 4). There was no significant difference between the baseline clinical parameters of the treated teeth in both groups indicating that both groups were balanced in disease distribution. The time taken to perform the procedure was also controlled equally among all participants. This split mouth study was designed to control for inter and intra patient difference in pain perception. Thus, the difference in inter patient pain perception affected the two treatment groups in the same way [11].

The present study indicates that the use of slimline-styled ultrasonic scaler tips for supragingival calculus removal may result in reducing pain 
sensations compared with conventional ultrasonic devices. Considering the overall aim to deliver dental care with a minimum of patient discomfort, it thus might be possible to increase the patient's compliance during dental treatment.

\section{CONCLUSION}

Within the limitations of this pilot study, it has been shown that Perio Slim PS scaler tip caused less pain and discomfort during scaling treatment when compared with conventional scaler tip.

\section{ACKNOWLEDGEMENT}

The authors would like to thank the staff from the Periodontology clinic, Faculty of Dentistry, University of Malaya for providing assistance during the clinical intervention.

\section{DECLARATION OF INTEREST}

The authors report no conflicts of interest.

\section{REFERENCES}

1. Friskopp, J., \& Hammarström, L. A comparative, scanning electron microscopic study of supragingival and subgingival calculus. Journal of Periodontology, 1980; 51(10), 553-562.

2. Lang, J., Karring, T., \& Araùjo, M. Anatomy of the periodontium. In Clinical Periodontology and Implant Dentistry. 2003. Blackwell Publishing Ltd.

3. Davies, R.M., Ellwood, R. P., Volpe, A. R., \& Petrone, M. E. Supragingival calculus and periodontal disease. Periodontology 2000, 1997; 15(1), 74-83.

4. Kleinknecht, R. A., Klepac R. K., \& Alexander L. D. Origins and characteristics of fear of dentistry. The Journal of the American Dental Association, 1973; 86(4), 842-848.

5. Baehni, P., Thilo, B., Chapuis, B., \& Pernet, D. Effects of ultrasonic and sonic sealers on dental plaque microflora in vitro and in vivo. Journal of Clinical Periodontology, 1992; 19(7), 455-459.

6. Arabaci, T., Cicek, Y., \& Canakci, C. F. Sonic and ultrasonic scalers in periodontal treatment: a review. International Journal of Dental Hygiene, 2007; 5(1), 2-12.

7. Pecheva, E., Sammons, R. L., Walmsley, A. D. The performance characteristics of a piezoelectric ultrasonic dental scaler. Medical Engineering \& Physics, 2016; 38(2), 199-203.

8. Thurnheer, T., Rohrer, E., Belibasakis, G. N., Attin, T., \& Schmidlin, P. R. Static biofilm removal around ultrasonic tips in vitro. Clinical Oral Investigations, 2014; 18(7), 1779-1784.

9. Bless, K. L., Sener, B., Dual, J., Attin, T., \& Schmidlin, P. R. Cleaning ability and induced dentin loss of a magnetostrictive ultrasonic instrument at different power settings. Clinical Oral Investigations, 2011; 15(2), 241-248.

10. Nardi, G. M., Di Giorgio, R., \& Sabatini, S. Effectiveness of tips for delicate micro-ultrasonic root planing comparing to tips for traditional ultrasonic root planing. Annali di Stomatologia, 2012; 3(3-4), 90.

11. Braun, A., Jepsen, S., Deimling, D., \& Ratka-Krüger, P. Subjective intensity of pain during supportive periodontal treatment using a sonic scaler or an Er: YAG laser. Journal of Clinical Periodontology, 2010; 37(4), 340-345.

12. Kocher T, Rodemerk B, Fanghänel J, Meissner G. Pain during prophylaxis treatment elicited by two power-driven instruments. Journal of Clinical Periodontology, 2005; 32(5), 535-538.

13. Pattison, A. M., Matsuda, S. A., \& Pattison, G. L. Clinical observations on ultrasonic tip selection for initial periodontal therapy.

14. Muhney, K. A., \& Dechow, P. C. Patients' perception of pain during ultrasonic debridement: a comparison between piezoelectric and magnetostrictive scalers. American Dental Hygienists Association, 2010; 84(4), 185-189.

15. Jepsen, S., Ayna, M., Hedderich, J., Eberhard, J. Significant influence of scaler tip design on root substance loss resulting from ultrasonic scaling: a laserprofilometric in vitro study. Journal of Clinical Periodontology, 2004; 31(11), 1003-1006.

16. Braun, A., Jepsen, S., \& Krause, F. Subjective intensity of pain during ultrasonic supragingival calculus removal. Journal of Clinical Periodontology, 2007;34(8), 668-672.

License Information: This work is licensed under a Creative Commons Attribution 